\title{
Using a UAV to Teach Undergraduates Math and Aircraft Performance
}

\section{Dr. Chadia Affane Aji, Tuskegee University}

Dr. Chadia Affane Aji is an associate professor in the Department of Mathematics in the College of Arts and Sciences at Tuskegee University. Dr. Aji's research interests lie in the areas of numerical analysis, computational applied mathematics, nonlinear partial differential equations, complex analysis, and on improving student learning in undergraduate mathematics courses. Dr. Aji has been a principle investigator or co-principle investigator on numerous interesting and engaging NSF projects. Dr. Aji is also very involved in retention activities at Tuskegee. In particular, she works closely with sources on campus to design strategies to assist incoming freshmen cope with first year mathematics classes. Dr. Aji is the recipient of the Tuskegee University Outstanding Faculty Performance Award for Research in 2010 from the College of Liberal Arts and Education.

\section{Dr. M. Javed Khan, Tuskegee University}

Dr. M. Javed Khan is professor and head of the Department of Aerospace Science Engineering at Tuskegee University. He received his Ph.D. in Aerospace Engineering from Texas A\&M University, his M.S. in Aeronautical Engineering from the U.S. Air Force Institute of Technology and his B.E. in Aerospace Engineering from the PAF College of Aeronautical Engineering, Pakistan. His research interests include aircraft design, experimental aerodynamics, and engineering education. 


\title{
Using a UAV to Teach Undergraduates Math and Aircraft Performance
}

\begin{abstract}
Hands-on activities based pedagogies have been determined to be effective in motivating and enhancing understanding of complex engineering concepts in undergraduate education. The use of laboratory exercises supporting theoretical concepts in the classroom is one such example of using hands-on activities. The Mathematics and Aerospace Science Engineering Departments are developing the capability to teach various concepts to undergraduate students using real-time data telemetered from an Unmanned Aerial Vehicle (UAV). This National Science Foundation (NSF) funded project uses a commercial-off the-shelf (COTS) UAV. The students develop a flight plan and then fly the UAV and collect data. The data is then analyzed and compared with the concepts learned in the classroom. This team of Math and Aerospace majors is also developing a low cost ( \$500) platform using commercial off-the-shelf components. The activity provides valuable hands-on teamwork experiences to the interdisciplinary team of students. Data on the effectiveness of the approach to gain these experiences for the students participating in the project are also presented. The paper details the two teaching modules that have been developed for the Pre-Calculus and Calculus courses and the Aircraft Performance course. The low-cost UAV was used to demonstrate the level turn module.
\end{abstract}

Keywords: Unmanned Aerial Vehicles (UAVs), Calculus, Aircraft Performance, Telemetry

\section{Introduction}

It has been observed consistently that an environment in which students are passive participants is less conducive to learning than an environment in which students are actively engaged. Techniques such as cooperative learning, collaborative learning, experiential learning, problembased learning and active learning, also referred to as inductive learning have been extensively researched. While the results of these various pedagogies vary, in general, their effect on student learning has been demonstrated to be positive in comparison to traditional lecturing. A number of active-learning approaches have been used to improve the learning of Science, Technology, Engineering Mathematics (STEM) concepts. These range from hands-on experiences using daily objects to web-based simulations and robotics. Most of the lessons in these active-learning experiences are based on connections with real life examples. A common theme has been 'amusement park physics' for web-based simulations teaching concepts of potential energy, kinetic energy, and centripetal/centrifugal forces. Others have used biological context such as fish and sperm locomotion to teach concepts of propulsion. Improvements in performance on an evaluation instrument for force-motion concepts have been demonstrated through the use of a micro-computer based laboratory curricula. Similarly, robot kits such as the LEGO-Mindstorm have been used for an active learning environment of Science Technology Engineering and Mathematics (STEM). 
A hands-on approach to learning concepts of STEM disciplines is therefore not a new pedagogical approach and has found wide usage, and enhanced learning has been reported in the literature. This aspect has been acknowledged by professional societies who have supported its adoption. Thus, while engineering education over the years has emphasized capstone design activities, more recently aerospace engineering curricula have incorporated elements of building and flying along with design due to active support from professional organizations such as the $\mathrm{AIAA}^{1}$ and $\mathrm{SAE}^{2}$ through their design/build/fly (DBF) competitions. These competitions are excellent venues for engineering students to practice their engineering knowledge and skills. Participation in these competitions requires considerable investment of time and financial resources and is therefore limited to schools with larger programs ${ }^{3}$. The DBF activity may not always yield successful results ${ }^{4}$ having possible negative impact on learning, motivation and self efficacy. The importance of success has been cited as the basis of the design-build-fly (DBF) experience in the Unified Engineering sequence at MIT $^{5}$. A number of examples are available where the DBF approach has been used to enhance learning of engineering concepts ${ }^{6,7,8,9}$. However, an important element missing in this 'product' development cycle is that of testing. While some design teams do conduct flight testing to varying degrees to determine various flight and operational characteristics but perhaps a formal flight-testing phase would provide much more information about the design, its strengths and weaknesses.

While availability of low-cost PC-based flight simulation software is a useful resource to some degree to complement the learning of various aspects of aircraft performance, stability \& control $^{10,11 ., 12}$ in programs not having the luxury of a flying laboratory, it provides a limited environment for gaining experience in essential skills to function in a real-world engineering environment. It is therefore felt that a low cost approach to providing undergraduate students with opportunity to collect and analyze real life data would be have a positive impact on students motivation and learning. The use of sub-scale models for concept validations and data collection as part of a flight vehicle design and development program is an approach used by industry ${ }^{13}$. Thus the development of learning opportunities based on such a platform is considered to be a viable approach.

This approach is not only beneficial for aerospace engineering concepts but also for students studying mathematics. Fundamental concepts in basic math courses are more often perceived by students as "required" rather than as key concepts and skills to open doors and empower them to pursue their education and careers in STEM disciplines. Anytime theoretical concepts are linked to examples of their application and when the students have to implement those concepts to make something that works, the reasoning (or concepts) is better understood and retained. This approach is well documented in literature on learning as problem-based learning or active learning ${ }^{14}$. While teachers may know or envision the long-term benefits of the topics or concepts being taught, immediate illustrations of the usefulness of the concepts and skills strengthen the students' understanding. Concepts taught on a need base, rather than on some established premise that mathematics is necessary for everything will result in far better learning outcomes. Engaging mathematics students on real-world problems such as the current approach being implemented will help them gain an intuitive and practical understanding of mathematics. In addition, students will enhance and strengthen their computational skills to better analyze and visualize the obtained data. 


\section{Objective}

This paper outlines the approach that is being developed and implemented at Tuskegee University to enhance the learning outcomes of undergraduate students in aerospace engineering and mathematics using real life flight data obtained from low cost commercial off the shelf (COTS) Unmanned Aerial Vehicles (UAVs) and locally integrated low-cost UAVs.

\section{Approach}

The use of UAVs to provide learning opportunities to undergraduate students in Math and Aerospace is a component of an NSF CRI Grant CNS 0855155: ATTRACT: Aerial and Terrestrial Testbed for Research in Aerospace, Computing, and maThematics. The grant supported the procurement of flight platforms and developing instructional materials. A two-tiered approach has been adopted. A commercial-off-shelf (COTS) aerial platform, fully integrated with an autopilot, telemetry system and graphical user interface (Fig. 1) has been procured. This UAV has a flying wing design and

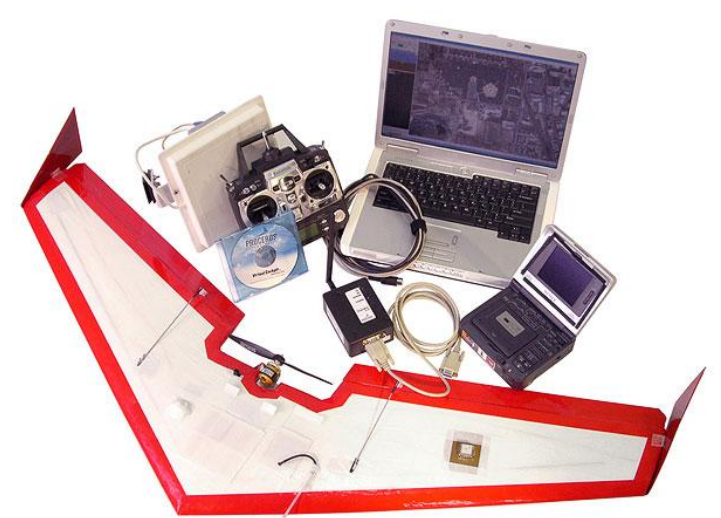

Figure 1: The Procerus 'Kestral' UAV is equipped with an autopilot (Kestral) with rate gyros, a global positioning system (GPS), altitude and speed sensors (pitot-static), and a telemetry modem for uplink and downlink communication. The flight data and position of the UAV is telemetered in real time to the ground station which consists of a transceiver and a laptop computer running the virtual cockpit software. Several upper division students from Math and Aerospace Engineering assisting with the project have been trained to fly the UAV in radio control as well as autopilot mode by pre-defining a flight path (Fig. 2). Considering the cost of using a COTS, fully integrated UAV, a more affordable approach is also being developed. This approach is to integrate commercial off the shelf components and software to build several low cost UAVs.

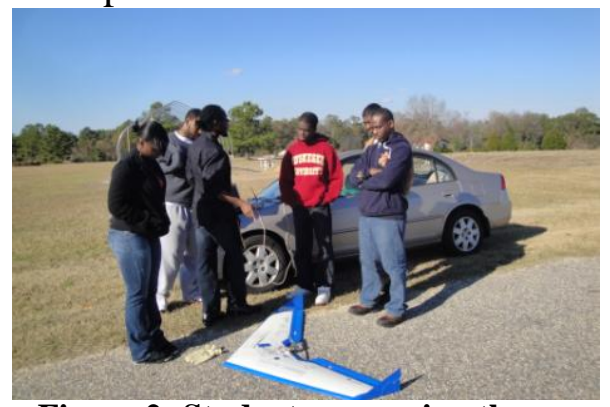

Figure 2: Students preparing the UAV for a flight test
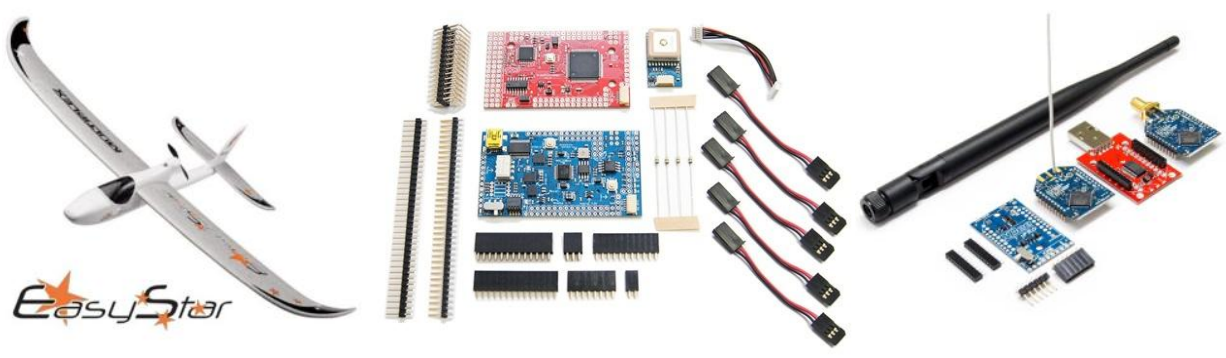

Figure 3: Low-cost UAV Components 
For this purpose the Arduino-based autopilot, and XBee or 3D Robotics telemetry hardware is integrated with an ARF (almost-ready to fly) airframe (Fig. 3). Open source software including the GUI is used. The details of this hardware and software are available www.diydrones.com. Of course there are some disadvantages of the low cost approach including lower endurance $(\sim 5 \mathrm{~min})$ and susceptibility to winds.

\section{Results and Discussion}

The math and aerospace students who were involved in the development of the teaching modules, learning to fly the COTS UAV and integrating the low-cost UAV experienced real life interdisciplinary teamwork (Fig. 4). They recognized the need to overcome challenges such as working with tools, software, and vocabulary. The math students enhanced their understanding of engineering concepts such as

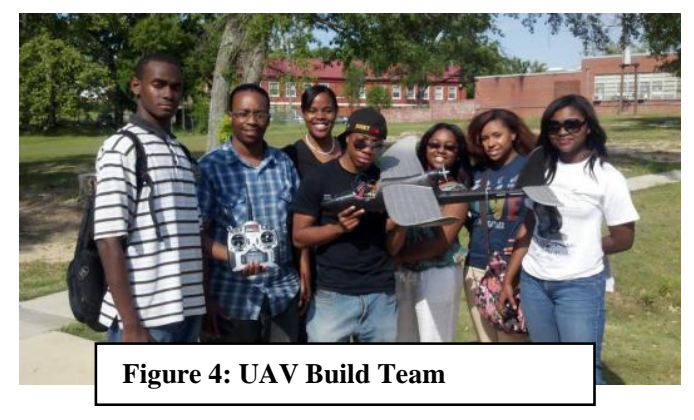
measurement of airspeed and aircraft stability. The students also improved their communication skills as they were to report their work as written technical procedures and presentations. The students were administered a survey (Fig. 5) to gage the efficacy of their hands-on experiences. The survey had 6 questions with response choices ranging from strongly agree to strongly disagree (a Likert 5-point scale). There was an opportunity to provide any additional comments about their experience. Nine of a total of 10 students ( 5 females and 5 males), who worked (are working) on the project responded to the survey. While none of these 9 students had any previous experience with UAVs, one student had prior experience with a surface robotic vehicle in high school. The survey results are shown in Fig. 5. As can be observed all the respondents either agreed or strongly agreed to the statements in the survey indicating that the project was a positive learning experience for students.

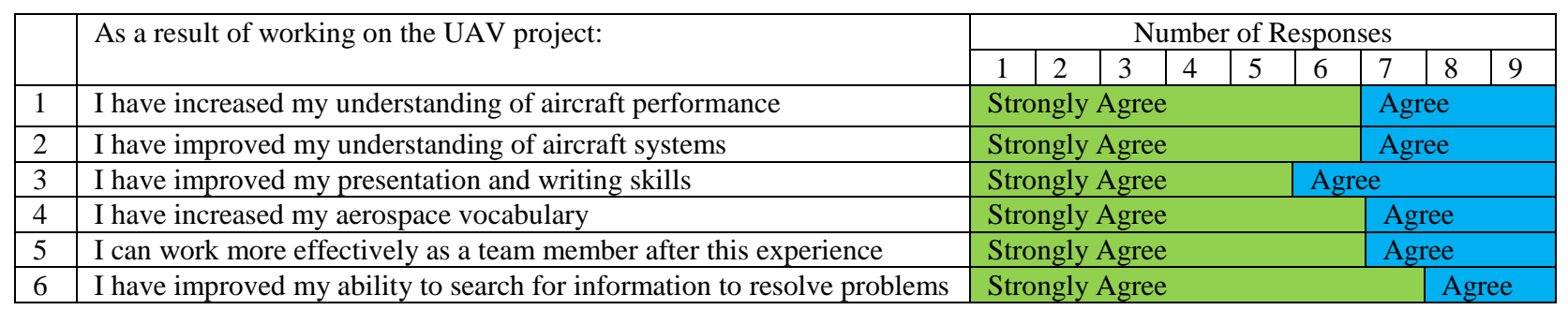

Figure 5: Survey Questions and Responses

Some of the typical comments from the students are:

"This project really got me into circuits and systems, I started working on my own arduino projects"

"During my internship interviews whenever I talked about this project, it always resulted in great interest, questions and discussion"

"I think project has greatly increased my research skills as well as presentation and writing (formulating scientific research papers)"

The team has developed several teaching modules and pilot tested one module in the aircraft performance course. The objectives of these teaching modules are: 
(a) Learn data import into MATLAB from a text file. The flight data telemetered by the UAV is saved as a text file and therefore needs to be preprocessed for analysis.

(b) Learn data manipulation in MATLAB to determine various flight performance parameters

(c) Learn data visualization in MATLAB by plotting the flight data in 2D as well as 3D.

(d) Determine flight performance parameters from flight data.

The following UAV-based teaching modules have been developed for use in the Aircraft Performance course and Pre-Calculus and Calculus courses.

(a) Level $360^{\circ}$ turn: This module provides a hands-on opportunity to fly the UAV in the prescribed circular path. The students analyze the telemetry flight data of the speed of the UAV and the bank angle to understand the impact of these two flight parameters on the radius of turn.

(b) Drag Polar Determination: This module allows the students to determine the drag polar of the aircraft. The rate of descent technique is used to calculate the data. The flight data when plotted provide the zero-lift drag $\left(\mathrm{C}_{\mathrm{Do}}\right)$ and lift-induced drag $\left(\mathrm{C}_{\mathrm{Di}}\right)$. The students can then compare the experimental data with the theoretical induced drag prediction by using say elliptic lift assumption. In the process the students learn how to calculate lift coefficient, aspect ratio etc. for the UAV. The module is planned for implementation during the Fall 2013 offering of the aircraft performance course.

The level-turn module (see Appendix) was pilot tested in the Aircraft Performance course last fall. While no formal evaluation was conducted due to the pilot nature of the implementation, highly positive informal anecdotal responses of the students taking the course were noted. The hands-on experience of planning the flight using the 'Virtual Cockpit' GUI, preparing the UAV for flight, the flight itself and data collection and learning about the changes in the bank angle by the autopilot to maintain a constant radius of angle necessitated by changes in airspeed (due to wind) was viewed by the students as an exciting activity and learning experience. The experience with the pilot testing has helped improve the module e.g. data reduction algorithms to convert GPS-based latitude and longitude of the UAV into Cartesian coordinates for ease of further analysis.

In Fall 2012, Tuskegee University mathematics students, who were taking Algebra course (Math 107) and Pre-Calculus course (Math 110) with the first author, were introduced to word problems related to the level turn module. These practice problems helped students to understand the connection between mathematics and physics. They identified the given quantities and the unknown in the word problems. They understood how to use the appropriate equation to solve for the unknown. Students also had to make sure about the units used and how these units made sense in the equation. Some practice questions are attached below. The hands-on activity will be used in the Spring 2013 with students in Calculus course and will be formally evaluated. After test flying the UAV, the telemeter flight data will be then recorded in a text file. Calculus students will then learn how to import these data in MATLAB. Therefore, Calculus students will have the opportunity to learn how to use MATLAB to import data from a text file and analyze it using $2 \mathrm{D}$ and/or 3D plots. A survey will be then given to check how the module can be helpful and beneficial to students. This module will also be used in the Fall 2013 offering of Aircraft 
Performance course and students' perceptions as well as impact on learning outcomes will be determined.

\section{Conclusion}

The hands-on activity using a UAV is a viable approach to improve student motivation, learning outcomes. The activity will be incorporated as a routinely used component of the aircraft performance course. The approach is cost effective and can be easily replicated. The experience of planning the flight using the 'Virtual Cockpit' GUI, preparing the UAV for flight, the flight itself and data collection and learning about the changes in the bank angle by the autopilot to maintain a constant radius of angle necessitated by changes in airspeed (due to wind) was viewed by the students as an exciting activity and learning experience. Assembling a UAV from off the shelf components, systems integration, and flight testing by the Math and Aerospace students was a valuable hands-on experience. This experience exposed the students to interdisciplinary teamwork.

\section{References}

1. http://www.aiaadbf.org/

2. http://students.sae.org/competitions/aerodesign/about.htm

3. Teaching for Success; Molding Course Syllabi to Support Student Capstone Design Work, Brian J. Novoselich and Justin Highley,

http://www.asee.org/activities/organizations/zones/proceedings/zone1/2008/Professional/ASEE12008_0118_paper.p df

4. Experiences using Flying Models in Competitions and Coursework, Robert P. Hansen, Lyle N. Long/, Todd A. Dellert, http://www.personal.psu.edu/lnl/papers/asee_region12.pdf

5. Design and Implementation of an Aeronautical Design-Build-Fly Course, Peter W. Young, Olivier L. de Weck, and Charles P. Coleman,

http://strategic.mit.edu/PDF_archive/3RefereedConference/3_22_ASEE_2003_868.pdf

6. UAV design Activities in a University Environment, Wong, K. C., 9th Australian International Aerospace

Congress, Canberra, Australia, 6-8 March 2001

7. The Development of Unmanned Aircraft Design in RMRL/NCKU, Fei-Bin Hsiao and Meng-Tse Lee, 4th Pacific International Conference on Aerospace Science and Technology, Kaohsiung, Taiwan, May 2001

8. System Engineering and Practice in Aircraft Design for Aerospace Education Fei-Bin Hsiao and Meng-Tse Lee, 4th UICEE Annual Conference on Engineering Education, Bangkok, Thailand, 7-10 February 2001

9. Using Design, Build, and Test Projects to Teach Engineering, Elger, D.F.; Beyerlein, S.W.; Budwig, R.S., Frontiers in Education 30th Annual Conference, 2000, Volume 2, Issue, 2000 Page(s):F3C/9 - F3C13

10. Virtual Flight Tests: An Effective Pedagogical Tool, M. Javed Khan and Bruce Heath, ASEE Annual

Conference, 11 -13 June, San Antonio, TX, 2012 
11. The Development and Implementation of a Flight Simulation Based Environment for Teaching Math \& Science, M. Javed Khan, Marcia Rossi, Chadia Aji, Bruce Heath, Proceedings of the SITE Conference, 5 - 9 March, 2012, Austin, TX

12. Teaching of Math and Physics Using a Flight Simulator, M. Javed Khan, Chadia Aji, Marcia Rossi, Bruce Heath, Proceedings of the ASEE SE Conference, 1 - 3 April, Starkville, MS

13. X-48B Flight-Test Progress Overview, Tim Risch, Gary Cosentino, and Christopher D. Regan, AIAA 2009-934, 47th AIAA Aerospace Sciences Meeting Including The New Horizons Forum and Aerospace Exposition, 5 - 8

January 2009, Orlando, Florida

14. National Research Council (2000), How People Learn. National Academy Press. 


\section{APPENDIX}

\section{Level Turn Module}

In this lesson we will determine the relationship between three flight parameters of the aircraft namely speed, bank angle and radius of turn for a coordinated level turn flight maneuver. We will solve several problems to determine one of three parameters when the other two are given. We will then verify our theoretical calculations by first flying a virtual flight test using the flight simulation environment collecting and analyzing data and then flying an Uninhabited Aerial Vehicle (UAV) and analyzing the telemetry data.

\section{Theoretical Background}

When the airplane is flying straight with wings level, the weight (W) of the aircraft is equal to the Lift (L) being generated (see figure below). When an object in motion has a circular path, it is subjected to a force in the direction of the radius but away from the center of the path. This force is called the centrifugal force. So when an aircraft is in a level turn the aircraft wing must produce lift (L) which should not only balance the weight of the airplane but also this centrifugal force which is trying to throw the airplane out of the turn. Note from the figure below that when an aircraft executes a level turn maneuver, the aircraft banks its wings, and now the lift vector is not vertical but at an angle $\Phi$ (the bank angle) from the vertical.

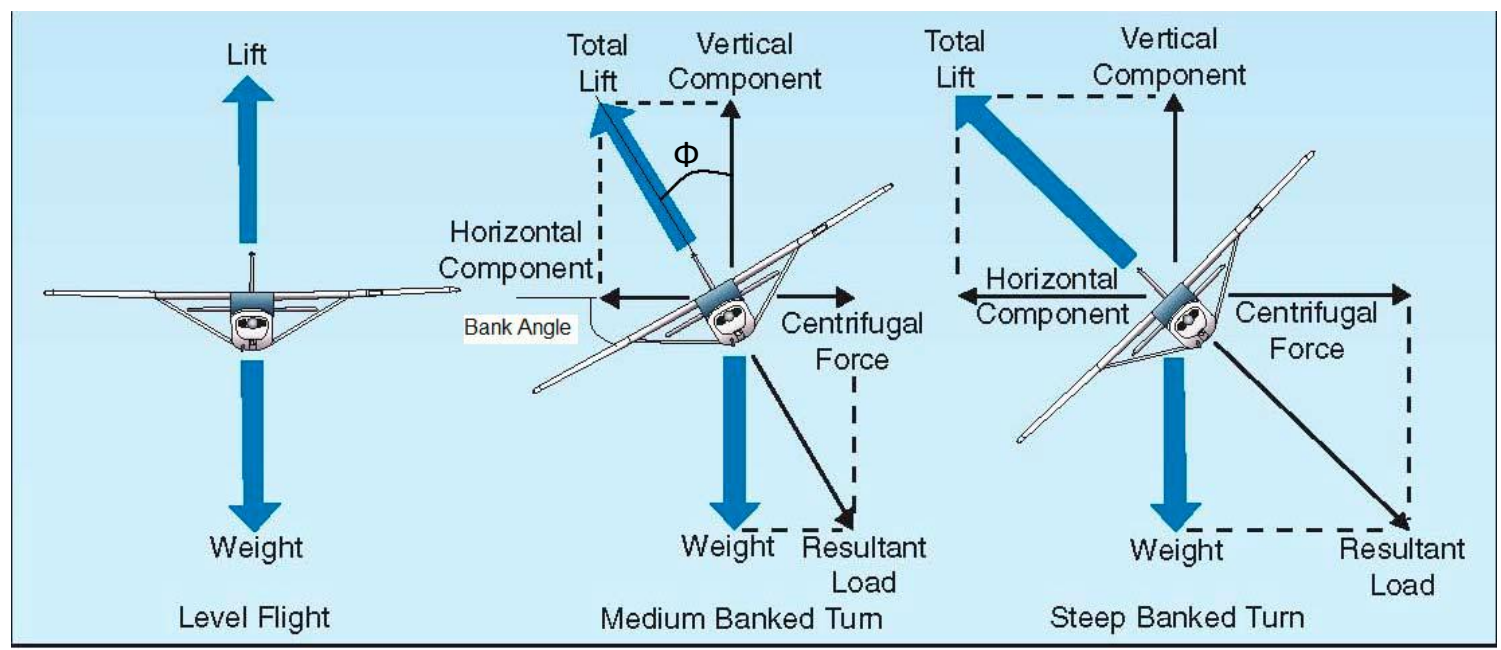

(From http://www.free-online-private-pilot-ground-school.com/Aerodynamics_in_flight.html)

Thus the centrifugal force $\mathrm{mV}^{2} / \mathrm{R}$ (where $\mathrm{m}=$ mass of the airplane, $\mathrm{V}=$ velocity of the airplane and $\mathrm{R}=$ radius of turn) is balanced by the horizontal component of lift LSin $\Phi$; and the weight $\mathrm{W}$ $=\mathrm{mg}$ is balanced by the vertical component of lift $\operatorname{LCos} \Phi$, where $\Phi$ is the bank angle. So, 


$$
\begin{aligned}
& \frac{m V^{2}}{R}=L \sin \Phi \\
& W=L \cos \Phi \quad \text { and } \quad \frac{L}{W}=\frac{1}{\cos \Phi}=n \quad \text { (load factor) } \\
& \text { also } W=m g=L \cos \Phi \\
& \text { so } m=\frac{L}{g} \cos \Phi \\
& \left(\frac{L}{g} \cos \Phi\right) V^{2} \\
& \text { and so } \frac{g}{R}=L \sin \Phi \\
& \text { or } \\
& R=\frac{V^{2} \cos \Phi}{g \sin \Phi} \\
& =\frac{V^{2} \cos \Phi}{g \sqrt{1-\cos ^{2} \Phi}} \\
& \text { and finally the radius of turn is given by } \\
& R=\frac{V^{2}}{g \sqrt{n^{2}-1}}
\end{aligned}
$$

Note that the radius of turn depends only on the velocity (which in this case is the true air speed) and the bank angle.

\section{Example Problem:}

An airplane is flying at 100 knots true air speed (TAS). If the airplane is required to make a turn with a radius of $2000 \mathrm{ft}$, what should be the bank angle?

Solution

The bank angle is related to the load factor by the relation $n=\frac{1}{\operatorname{Cos} \phi}$ and since $R=\frac{V^{2}}{g \sqrt{n^{2}-1}}$ then $R^{2}=\frac{V^{4}}{g^{2}\left(n^{2}-1\right)}$ or $n=\left(1+\frac{V^{4}}{g^{2} R^{2}}\right)^{1 / 2}$.

Now $1 \mathrm{knot}=1$ nautical mile $/$ hour $=1.1515 \mathrm{mile} / \mathrm{hour}=(1.515)(88) \mathrm{ft} / \mathrm{min}=$ $(1.5150(88)(1 / 60) \mathrm{ft} / \mathrm{sec}=1.69 \mathrm{ft} / \mathrm{sec}$. So, $100 \mathrm{knots}=(1.69)(100)=169 \mathrm{ft} / \mathrm{sec}$ and $\mathrm{g}=32.2$ $\mathrm{ft} / \mathrm{sec}^{2}$. 
Therefore, for a radius $\mathrm{R}=2000 \mathrm{ft}, n=\left(1+\frac{(169)^{4}}{(32.2)^{2} \times(2000)^{2}}\right)$ or $\quad n=1.094$.

And since $\mathrm{n}=1 / \operatorname{Cos} \Phi$, that is $\operatorname{Cos} \Phi=1 / \mathrm{n}$. Hence, $\Phi=\cos ^{-1}(1 / 1.26)=23.92^{\circ}$.

\section{PRACTICE PROBLEMS}

1. Plot bank angle vs. load factor for $0^{\circ}<\Phi<90^{\circ}$.

2. Plot radius of turn vs. bank angle for various speeds.

3. An airplane is required to maintain a radius of turn of $500 \mathrm{ft}$ while flying at a TAS of 75 knots. What bank angle should the pilot maintain? (Ans: $45^{\circ}$ )

4. An airplane flying at a TAS of 275 knots is executing a level turn with a bank angle of $30^{\circ}$. What is its radius of turn? (Ans: $9602 \mathrm{ft}$ )

5. If the weight of an airplane is $1500 \mathrm{lbs}$ and has a wing reference area $(\mathrm{S})$ of $175 \mathrm{ft}^{2}$ and the maximum lift coefficient $\left(\mathrm{C}_{\mathrm{Lmax}}\right)$ of the airplane is 2.25 , can it execute a radius of turn of $500 \mathrm{ft}$ while flying at a TAS of 75 knots?

\section{Flight Test}

A UAV will be used for the flight test. This UAV is equipped with an autopilot with rate gyros, a global positioning system (GPS), altitude and speed sensors (pitot-static) and a telemetry modem for uplink and downlink communication. The flight data and position of the UAV is telemetered in real time to the ground station, which consists of a transceiver and a laptop computer running the virtual cockpit software.

The flight test will consist of following:

- Take-off and climb to a pre-determined altitude

- Loiter over pre-designated take-off point (several orbits) at a pre-determined speed and radius

- Fly to a designated Rally point at a pre-determined altitude and fly several orbits at a predetermined speed and radius of turn

- Land at the designated location 
You will then import the data into Matlab and perform the analysis to determine the radius of turn. The results from the flight test will then be compared with the theoretical solution and appropriate observations made.

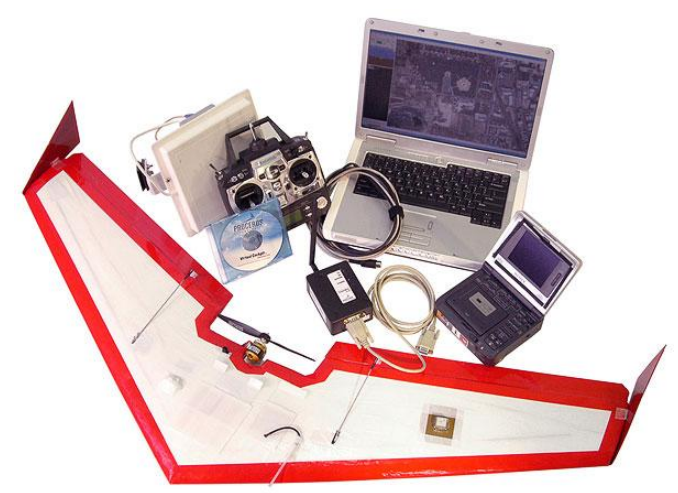

\section{UAV Telemetry Data File}

The telemetry data is saved as a text file. A typical data file is shown below.

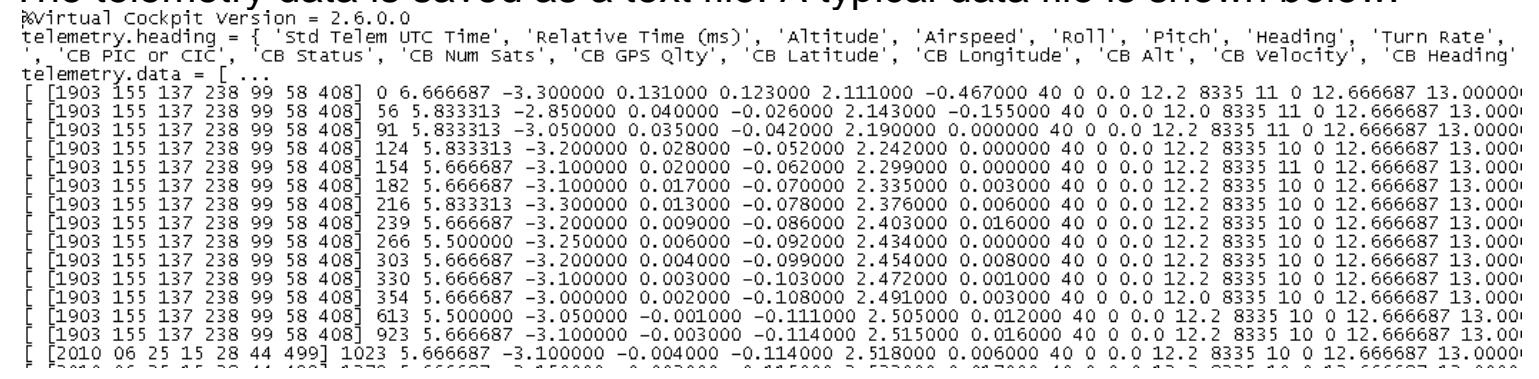

In order to process this data e.g. for plotting, export it to MATLAB. Students will be assisted on how to use MATLAB.

\section{Drag Polar Determination Module}

The drag of an aircraft consists of several parts. The two important components that contribute to the total drag of the aircraft are:

Friction Drag: This is the result of the viscosity of the air. The smoother the exposed surface of the aircraft, the smaller this drag will be.

Induced Drag: This is the result of the downwash that is produced by the wing tip vortices. These vortices are produced due the pressure difference between the top and bottom surfaces of the wing. Thus, whenever a wing is producing lift, it will also produce drag; and the higher the lift, the larger the induced drag will be.

Other phenomena, which contribute to the drag, are separated flows, protuberances, shock waves (during supersonic flow) etc.

The drag of an aircraft is usually given as a 'drag polar' by the relationship: 
$C_{D}=C_{D_{0}}+k C_{L}{ }^{2}$ where $C_{D_{0}}$ is the drag coefficient at zero lift and includes all components of drag (also known as parasite drag) except induced drag, and $k C_{L}{ }^{2}$ is the drag due to lift or induced drag. Here $k=\frac{1}{\pi \in A R}$ where $\mathrm{e}=$ Oswald efficiency factor and $\mathrm{AR}=$ aspect ratio.

While there are various techniques of theoretically determining the drag of an aircraft, this is ultimately validated through flight tests. You will conduct a flight test using the Procerus UAV to determine its drag. Specifically you will determine the Oswald efficiency factor (e) and $\mathrm{C}_{\mathrm{Do}}$. This UAV has a flying wing design and is equipped with an autopilot (Kestral) with rate gyros, a global positioning system (GPS), altitude and speed sensors (pitot-static) and a telemetry modem for uplink and downlink communication. The flight data and position of the UAV is telemetered in real time to the ground station, which consists of a transceiver and a laptop computer running the virtual cockpit software.

\section{Theoretical Background}

The drag of the UAV will be determined by the 'glide path' method. In this method the rate of descent in a 'power-off' glide is measured and is then used to calculate the glide angle $\gamma$. This is then utilized to determine the lift and drag coefficients. The following equations are used:

Rate of Descent: $\dot{h}=\frac{\Delta h}{\Delta t}$; True Air Speed: $V=\frac{W_{i}}{\sigma}$, where $V_{i}=$ indicated airspeed and $\sigma=$ ratio of density at the altitude and the standard density at the altitude

Then $\operatorname{Sin} \gamma=\frac{\hbar}{V}$, and the lift coefficient $C_{L}=\frac{W \operatorname{Cos} Y}{q S}$, drag coefficient $C_{D}=\frac{W \operatorname{Sin} y}{q S}$

Once the $C_{L}$ and $C_{D}$ are determined for various true airspeeds and plotted, that ' $\mathrm{e}$ ' and $\mathrm{C}_{\mathrm{Do}}$ can be found. A typical plot of $C_{D} \vee C_{L}^{2}$ is given in the figure below. 


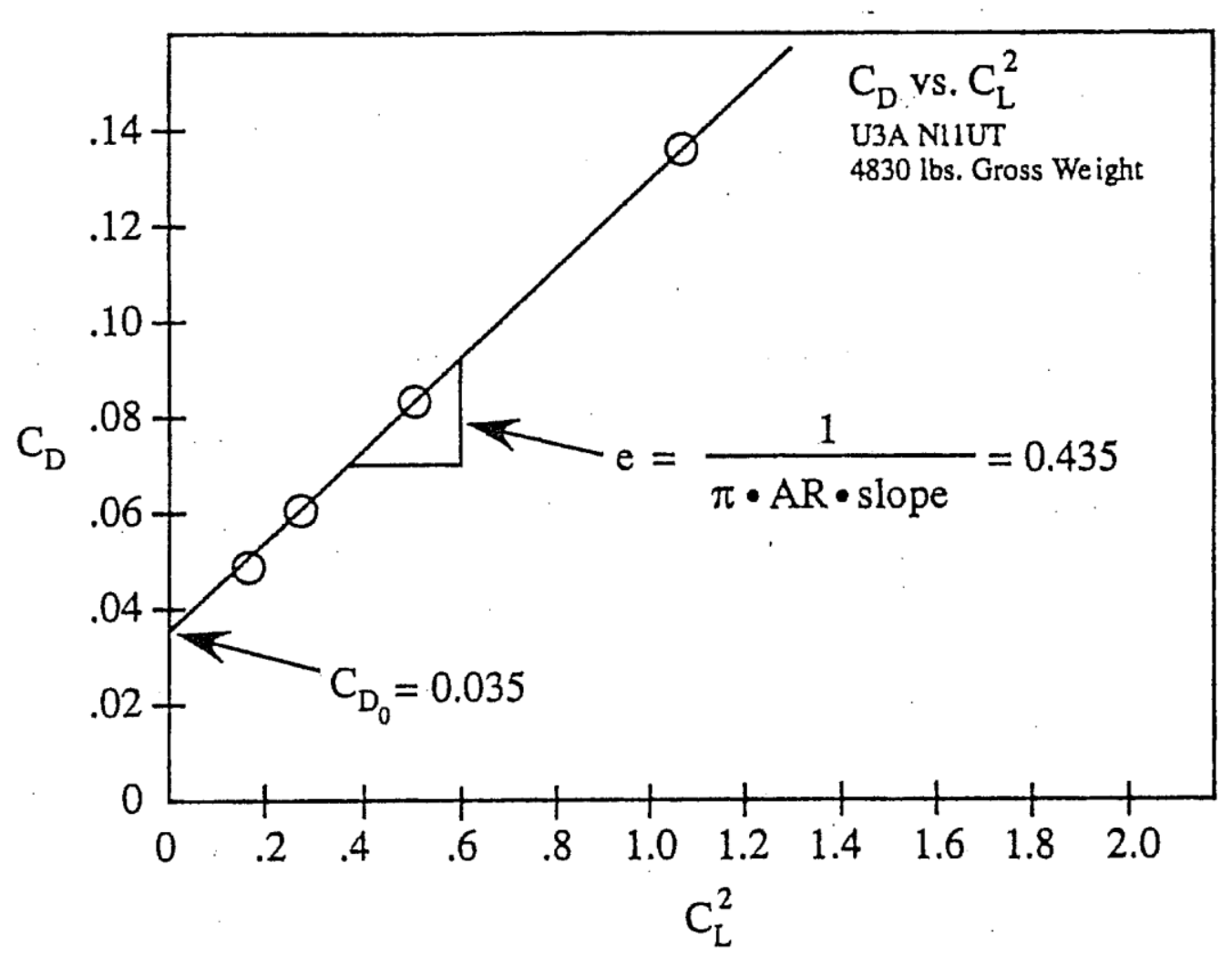

(From: Kimberlin, R., Flight Testing of Fixed-Wing Aircraft, American Institute of Aeronautics and Astronautics, 2003, page 163.)

\section{Flight Test}

The UAV will be flown to conduct the actual flight tests for determining its drag characteristics. The Procerus is a flying wing design with the ability to be flown on autopilot and can telemeter flight data. The procedure to fly the UAV is given its manuals.

\section{Procedure}

1. Establish a straight and level flight at a pre-determined speed and altitude.

2. Cut power to idle and establish a glide at a pre-determined speed for a predetermined time.

3. Advance power climb back to the previous altitude and establish straight and level flight.

4. Cut power to idle and establish a glide at another pre-determined speed for a predetermined time.

5. Repeat $3 \& 4$ for several different speeds.

6. Terminate the flight test and land the UAV.

7. Calculate the rate of descent for each speed using the telemetered data.

8. Calculate the glide angle $\gamma$ for each speed and associated rate of descent.

9. Calculate the lift and drag coefficients for each glide angle.

10. Plot $\mathrm{C}_{\mathrm{L}}^{2}$ vs $\mathrm{C}_{\mathrm{D}}$.

11. Determine $C_{\text {Do }}$ and e' from the plot. 